September 23, 1998 (corrected.)

UMN-TH-1626/98, TPI-MINN-98/11-T

hep-th/9808045

\title{
Complex Monopoles
}

\section{in the Georgi-Glashow-Chern-Simons Model}

\author{
Bayram Tekin, Kamran Saririan and Yutaka Hosotani \\ School of Physics and Astronomy, University of Minnesota \\ Minneapolis, MN 55455, U.S.A.
}

\begin{abstract}
We investigate the three dimensional Georgi-Glashow model with a Chern-Simons term. We find that there exist complex monopole solutions of finite action. They dominate the path integral and disorder the Higgs vacuum, but electric charges are not confined. Subtleties in the gauge fixing procedure in the path integral and issues related to Gribov copies are noted.
\end{abstract}

PACS: $11.10 . \mathrm{Kk} ; 11.15 . \mathrm{Kc} ; 11.15 . \mathrm{Tk}$

Keywords: Monopoles, Chern-Simons theory, Confinement 


\section{Introduction}

Years ago Polyakov showed that in the three-dimensional Georgi-Glashow model, or more generally in the compact $\mathrm{QED}_{3}$, monopole configurations in the Euclidean space make dominant contributions in the functional integral for the confinement of electric charges [1]. The logarithmic potential between two electric probe charges is converted to a linear potential in the background of a monopole gas, leading to the linear confinement. The Higgs vacuum is disordered. The expectation value of the triplet Higgs field vanishes $(\langle\vec{\phi}\rangle=0)$, whereas all components of the $S U(2)$ gauge fields acquire masses. The long range order in the Higgs vacuum is destroyed by monopole configurations.

Gauge theory in three dimensions can accommodate a purely topological term, the Chern-Simons term, which affects the equations of motion. It gives a topological mass to gauge fields [2]. In the Georgi-Glashow model, even in perturbation theory, the unbroken $U(1)$ gauge field also becomes massive, and thus the issue of the linear confinement disappears in the presence of the Chern-Simons term; there is no long-range force in the Georgi-Glashow-Chern-Simons (GGCS) model to start with. The electric flux is not conserved. It does not matter for the issue of the confinement whether or not monopole configurations dominate in the functional integral.

Once the Chern-Simons term is added the action becomes complex in the Euclidean space. Many authors have shown that there is no real monopole-type field configuration of a finite action which solves the Euler equations [3]-[7]. This fact has been interpreted as indicating the irrelevance of (real) monopole configurations in the model.

There remain a few puzzles. Although there is no confinement in the presence of the Chern-Simons term, there remains the issue of the long-range order in the Higgs vacuum. How can the Higgs vacuum be disordered if monopole configurations are totally irrelevant?

Does the expectation value $\langle\vec{\phi}\rangle$ become nonvanishing and the long-range order is restored once the Chern-Simons term is added? Also, if the theory allows complex monopole configurations, does their contribution to the partition function vanish as in the real case? There are also subtle questions related to the gauge invariance as well as Gribov copies in various gauges in the GGCS model which, to our knowledge, have not yet been answered. 
We shall re-examine these considerations in the context of complex monopole solutions to show that the Higgs vacuum remains disordered. Although there are no real monopole configurations which solve the Euler equations, there exist complex monopole configurations which extremize the Euclidean action in a fixed gauge. They dominate the functional integral in quantum theory and destroy the long-range order in the Higgs vacuum. The effect of Gribov copies is also re-examined. What we mean by complex monopoles will be clear in the text but for now we should state that these are complex-valued solutions to the equations of motion. The non-abelian field strengths are complex but the $U(1)$ 't Hooft field strengths are real and exactly those of a magnetic monopole. With the $U(1)$ projection our complex monopoles can be interpreted as the topological excitations characterized by the the group $\Pi_{2}(S O(3) / U(1))=Z$.

It is worthwhile to recall the correspondence between compact $\mathrm{QED}_{3}$ and the Josephson junction system in the superconductivity [8]. The normal barrier region sandwiched by two bulk superconductors becomes superconducting due to supercurrents flowing through the barrier. The three-dimensional compact QED is related to the Josephson junction system by the electro-magnetic dual transformation. The $U(1)$ field strengths $\left(E_{1}, E_{2}, B\right)$ in the Georgi-Glashow model correspond to $\left(B_{1}, B_{2}, E_{3}\right)$ in the Josephson junction. Electric charges in the Georgi-Glashow model are magnetic charges inserted in the barrier in the Josephson junction. If there were no supercurrents, the magnetic flux between a pair of magnetic monopole and anti-monopole inserted in the barrier forms dipole fields, giving a logarithmic potential between the pair. However, due to supercurrents the magnetic flux is squeezed to form a Nielsen-Olesen vortex giving rise to a linear potential.

Monopoles (instantons) in compact $\mathrm{QED}_{3}$ are supercurrents in the Josephson junction. Polyakov introduced a collective field $\chi$ which mediates interactions among monopoles. The field $\chi$ corresponds to the difference between the phases of the Ginzburg-Landau order parameters $\Psi_{\mathrm{GL}}$ in the bulk superconductors on both sides of the barrier in the Josephson junction. Both $\chi$ and $\delta\left(\arg \Psi_{\mathrm{GL}}\right)$ satisfy the same sine-Gordon type equation.

Now we add the Chern-Simons term in the Georgi-Glashow model. At the moment we haven't understood what kind of an additional interaction in the Josephson junction 
system corresponds to the Chern-Simons term in the Georgi-Glashow model. It could be a $\theta F_{\mu \nu} \tilde{F}^{\mu \nu}$ term in the superconductors on both sides. Normally a $\theta F_{\mu \nu} \tilde{F}^{\mu \nu}$ term is irrelevant in QED. However, if the values of $\theta$ on the left and right sides are different, this term may result in a physical consequence, which may mimic the effect of the Chern-Simons term in the Georgi-Glashow model.

If monopoles are irrelevant in the presence of the Chern-Simons term, it would imply that suppercurrents cease to flow across the barrier in the corresponding Josephson junction. Although we have not found the precise analogue in the Josephson system yet, and therefore we cannot say anything definite by analogy, we feel that it is very puzzling if suppercurrents suddenly stop to flow. Monopoles should remain important even in the presence of the Chern-Simons term. The correspondence between the Chern-Simons theory and the Josephson junction arrays has been discussed in ref. [9].

As we shall discuss below, there is a subtle issue in quantizing a Chern-Simons theory. The arguments below are based on the Chern-Simons theory in a fixed gauge. In the path integral a gauge condition restricts functional space to be integrated. Within this subspace complex monopole solutions are found. This is a delicate issue as the Chern-Simons term is not gauge invariant.

\section{The model}

The action for the scalar fields interacting with the gauge fields in the threedimensional Euclidean space is given by $S=S_{0}+S_{\mathrm{CS}}+S_{h}$ where

$$
\begin{aligned}
S_{0} & =-\frac{1}{2 g^{2}} \int d^{3} x \operatorname{tr}\left(F_{\mu \nu} F^{\mu \nu}\right) \\
S_{\mathrm{CS}} & =-\frac{i \kappa}{g^{2}} \int d^{3} x \epsilon^{\mu \nu \lambda} \operatorname{tr}\left(A_{\mu} \partial_{\nu} A_{\lambda}+\frac{2}{3} A_{\mu} A_{\nu} A_{\lambda}\right) \\
S_{h} & =\frac{1}{g^{2}} \int d^{3} x\left[\frac{1}{2}\left(D_{\mu} h^{a}\right)^{2}+\frac{\lambda}{4}\left(h^{a} h^{a}-v^{2}\right)^{2}\right]
\end{aligned}
$$

We adopt the notation $A_{\mu}=\frac{i}{2} A_{\mu}^{a} \tau^{a}, F_{\mu \nu}=\partial_{\mu} A_{\nu}-\partial_{\nu} A_{\mu}+\left[A_{\mu}, A_{\nu}\right], h=\frac{i}{2} h^{a} \tau^{a}$, and $D_{\mu} h=\partial_{\mu} h+\left[A_{\mu}, h\right]$. The classical equations of motion are

$$
D_{\mu} F^{\mu \nu}+\frac{i}{2} \kappa \epsilon^{\nu \lambda \mu} F_{\lambda \mu}=\left[h, D^{\nu} h\right]
$$




$$
D_{\mu} D^{\mu} h=-\lambda\left(\operatorname{tr} h^{2}+m^{2}\right) h
$$

Notice that $S_{\mathrm{CS}}$ is pure imaginary in the Euclidean space for real gauge field configurations. Equations of motion become complex. To define a quantum theory, one needs to fix a gauge. We would like to find dominant field configurations. Polyakov, in the theory where $\kappa=0$, showed that monopole configurations are essential in removing the degeneracy of the vacuum and lead to the linear confinement of electric charges. For $\kappa \neq 0$ a real monopole configuration is shown to have an infinite action which makes its contribution vanish in the vacuum. Pisarski [3] interpreted this as the confinement of monopole. Fradkin and Schaposnik [6] consider a theory where Chern-Simons term is induced as a one loop effect after integrating fermions. In this case the t'Hooft-Polyakov real monopole [1, 10] minimizes the classical equations of motion but not at the one loop level. Fradkin and Schaposnik deform the real t'Hooft-Polyakov monopole in the complex configuration space. They have concluded that a monopole-antimonopole pair is bound together by a linearly growing potential.

It is clear that more careful analysis is necessary to find the absence or existence of complex monopole configurations and their implications. In the context of path integrals, stationary points of the exponent in the integrand may be located generically at complex points, though the original integration over field configurations is defined along the real axis. In the saddle-point method for integration such saddle-points give dominant contributions in the integral. Complex monopoles can be vital in disordering the vacuum.

When $v \neq 0$ in (2.1), the perturbative vacuum manifold is $S O(3) / U(1)$. Following t'Hooft and Polyakov, we make the spherically symmetric monopole ansatz which breaks $S O(3)_{\text {gauge }} \times S O(3)_{\text {rotation }}$ to $S O(3)$.

$$
\begin{aligned}
& h^{a}(\vec{x})=\hat{x}^{a} h(r) \\
& A_{\mu}^{a}(\vec{x})=\frac{1}{r}\left[\epsilon_{a \mu \nu} \hat{x}^{\nu}\left(1-\phi_{1}\right)+\delta^{a \mu} \phi_{2}+\left(r A-\phi_{2}\right) \hat{x}^{a} \hat{x}_{\mu}\right]
\end{aligned}
$$

where $\hat{x}^{a}=x^{a} / r$. Field strengths are

$$
F_{\mu \nu}^{a}=\frac{1}{r^{2}} \epsilon_{\mu \nu b} \hat{x}^{a} \hat{x}^{b}\left(\phi_{1}^{2}+\phi_{2}^{2}-1\right)+\frac{1}{r}\left(\epsilon_{a \mu \nu}-\epsilon_{\mu \nu b} \hat{x}^{a} \hat{x}^{b}\right)\left(\phi_{1}^{\prime}+A \phi_{2}\right)
$$




$$
+\left(\delta^{a \nu} \hat{x}^{\mu}-\delta^{a \mu} \hat{x}^{\nu}\right) \frac{1}{r}\left(\phi_{2}^{\prime}-A \phi_{1}\right)
$$

Classical equations of motion are

$$
\frac{1}{r^{2}} \frac{d}{d r}\left(r^{2} h^{\prime}\right)-\lambda\left(h^{2}-v^{2}\right) h-\frac{2}{r^{2}}\left(\phi_{1}^{2}+\phi_{2}^{2}\right) h=0
$$

for the Higgs field and

$$
\begin{aligned}
& \phi_{1} \phi_{2}^{\prime}-\phi_{2} \phi_{1}^{\prime}-A\left(\phi_{1}^{2}+\phi_{2}^{2}\right)+\frac{i}{2} \kappa\left(1-\phi_{1}^{2}-\phi_{2}^{2}\right)=0 \\
& \left(\phi_{1}^{\prime}+A \phi_{2}\right)^{\prime}+A\left(\phi_{2}^{\prime}-A \phi_{1}\right)+\frac{\phi_{1}}{r^{2}}\left(1-\phi_{1}^{2}-\phi_{2}^{2}\right)+i \kappa\left(\phi_{2}^{\prime}-A \phi_{1}\right)-h^{2} \phi_{1}=0 \\
& \left(\phi_{2}^{\prime}-A \phi_{1}\right)^{\prime}-A\left(\phi_{1}^{\prime}+A \phi_{2}\right)+\frac{\phi_{2}}{r^{2}}\left(1-\phi_{1}^{2}-\phi_{2}^{2}\right)-i \kappa\left(\phi_{1}^{\prime}+A \phi_{2}\right)-h^{2} \phi_{2}=0
\end{aligned}
$$

for the gauge fields. Finite action solutions to these equations are necessarily complex for $\kappa \neq 0$. Note that it follows from Eqs. (2.7) and (2.8) that

$$
\phi_{1} \phi_{2}^{\prime}-\phi_{2} \phi_{1}^{\prime}-A\left(\phi_{1}^{2}+\phi_{2}^{2}\right)+\frac{i}{2} \kappa\left(1-\phi_{1}^{2}-\phi_{2}^{2}\right)=\mathrm{const}
$$

Eq. (2.6) gives an additional information that the constant in Eq. (2.9) is 0 .

However, due caution is necessary in writing down equations of motion in quantum theory. The Chern-Simons term is not gauge invariant. Even if the action is varied in a pure gauge direction, it may change. In other words the action need not be stationary under such variations. In the monopole ansatz one combination of Eqs. (2.6) - (2.8) corresponds to such variations. If one fixes the gauge first, this particular equation does not follow from the gauge-fixed action.

This becomes clearer when equations are derived from the action written in terms of the monopole ansatz. The action is then written as follows (using eq. (2.3)):

$$
\begin{aligned}
S=\frac{4 \pi}{g^{2}} \int_{0}^{\infty} d r & \left\{\left(\phi_{1}^{\prime}+A \phi_{2}\right)^{2}+\left(\phi_{2}^{\prime}-A \phi_{1}\right)^{2}+\frac{1}{2 r^{2}}\left(\phi_{1}^{2}+\phi_{2}^{2}-1\right)^{2}\right. \\
& +i \kappa\left[\phi_{1}^{\prime} \phi_{2}-\phi_{2}^{\prime}\left(\phi_{1}-1\right)+A\left(\phi_{1}^{2}+\phi_{2}^{2}-1\right)\right] \\
& \left.+\frac{r^{2}}{2} h^{\prime 2}+h^{2}\left(\phi_{1}^{2}+\phi_{2}^{2}\right)+\frac{\lambda r^{2}}{4}\left(h^{2}-v^{2}\right)^{2}\right\}
\end{aligned}
$$

The regularity at the origin and the finiteness of the action place boundary conditions

$$
\text { at } r=0: \quad h=0 \quad, \quad \phi_{1}=1 \quad, \quad \phi_{2}=0
$$




$$
\text { at } r=\infty: \quad h=v \quad, \quad \phi_{1}=0 \quad, \quad \phi_{2}=0 \quad, \quad A=0
$$

In the original 't Hooft-Polyakov [1, 10] monopole solution, $\phi_{2}(r)=A(r)=0$.

For $v \neq 0$ the unbroken $U(1)$ is parametrized by residual gauge transformations generated by $\Omega=\exp \left\{\frac{i}{2} f(r) \hat{x}^{a} \sigma^{a}\right\}$ where $f(0)=0$. Under this gauge transformation the fields transform as

$$
\begin{aligned}
\phi_{1} & \rightarrow+\phi_{1} \cos f+\phi_{2} \sin f \\
\phi_{2} & \rightarrow-\phi_{1} \sin f+\phi_{2} \cos f \\
A & \rightarrow A-f^{\prime}
\end{aligned}
$$

The equations of motion are invariant under this transformation but the action (2.10) is not. Under a more general gauge transformation $A \rightarrow \Omega A \Omega^{-1}+g^{-1} \Omega d \Omega^{-1}$,

$$
S_{C S} \rightarrow S_{C S}+\frac{i \kappa}{g^{2}} \int \operatorname{tr} d\left(A \wedge d \Omega^{-1} \Omega\right)+\frac{i \kappa}{3 g^{2}} \int \operatorname{tr} d \Omega^{-1} \Omega \wedge d \Omega^{-1} \Omega \wedge d \Omega^{-1} \Omega
$$

If the theory is defined on $S^{3}$, the first term vanishes. The last term is the winding number of the mapping $\Omega$. This leads to the celebrated quantization condition $4 \pi \kappa / g^{2}=n=$ an integer [2].

On $R^{3}$, however, the first term does not vanish for monopole configurations. Under (2.12) the second and last terms in (2.13) are $\left(4 \pi i \kappa / g^{2}\right) \sin f(\infty)$ and $\left(4 \pi i \kappa / g^{2}\right)(f(\infty)-$ $\sin f(\infty))$, respectively. Hence

$$
S \rightarrow S+\frac{4 \pi i \kappa}{g^{2}} f(\infty)
$$

If the action is viewed as a functional of all $h, A, \phi_{1}$, and $\phi_{2}$ and varied with respect to those fields, then one would obtain Eqs. (2.5) - (2.8). It is obvious, however, that Eq. (2.6) and the boundary condition (2.11) are incompatible at $r=\infty$. There would be no solution of a finite action. Instead, one might fix a gauge first and vary the action within the gauge chosen. Indeed, this is what is done in quantum theory either in the canonical formalism or in the path integral formalism. In the path integral we start with

$$
Z=\int \mathcal{D} A \mathcal{D} h \Delta_{F}(A) \delta[F(A)] e^{-S}
$$


What we are looking for is a field configuration which extremizes $S$ within the gauge chosen; $\left.\delta S\right|_{F(A)=0}$. If the action is manifestly gauge invariant, the order of two operations, fixing a gauge and extremizing $I$, does not matter. However, in the presence of the Chern-Simons term, the action is not manifestly gauge invariant. As we shall see explicitly in the following section, two configurations $A^{(j)}(j=1,2)$ determined by $\left.\delta S\right|_{F_{j}(A)=0}$ are physically different in general, i.e. $A^{(1)}$ is not on a gauge orbit of $A^{(2)}$.

Before closing this section, we would like to remark that there are three approaches in defining the path integral. The issue is how to define the configuration space $A_{\mu}$ in (2.15). The first possibility is to restrict gauge orbits such that transformation function $\Omega$ be $S^{3}$-compatible, namely $\left.\Omega\right|_{r=\infty}=\Omega_{\infty}$. For $(2.12)$ it implies that $f(\infty)=2 \pi p$ where $p$ is an integer. With this $e^{-S}$ becomes gauge invariant. However, this restriction leads to conflict with gauge fixing. Suppose that a gauge configuration is given. Now one fixes a gauge. However, a gauge orbit of the given configuration may not intersect the gauge fixing condition. For instance, if $\int_{0}^{\infty} d r A \neq 2 \pi p$, then the configuration cannot be represented in the radial gauge. If $\phi_{2} /\left.\phi_{1}\right|_{r=\infty} \neq 0$, then the configuration cannot be represented in the unitary gauge. If there is no $f(r)$ satisfying

$$
f^{\prime \prime}+\frac{2}{r} f^{\prime}-\frac{2}{r^{2}}\left\{\phi_{1} \sin f+\phi_{2}(1-\cos f)\right\}=A^{\prime}+\frac{2 A}{r}-\frac{2 \phi_{2}}{r^{2}},
$$

then the configuration cannot be represented in the radiation gauge. Put it differently, the gauge fixing procedure removes a part of physical gauge configurations. This approach is not acceptable.

The second possibility is to impose no restriction on $\Omega$. In the case $e^{-S}$ is not gauge invariant in general. In this paper we adopt this approach to find consequences.

The third possibility is to restrict gauge field configurations $A_{\mu}$ to be $S^{3}$ compatible. This excludes monopole ansatz (2.3) entirely. With this restriction the total monopole charge must vanish. This is certainly a legitimate approach, and there occurs no problem of the gauge invariance of the theory. One has to address a question why the spacetime needs to be compactified from $R^{3}$ to $S^{3}$ in defining a theory. We leave this possibility for future consideration. 


\section{Gauge choices}

There are four gauge choices which are typically considered:

(i) Radial gauge $\left(A=\hat{x}^{a} \hat{x}^{\mu} A_{\mu}^{a}=0\right)$.

As is obvious from (2.12), this gauge choice is always possible. $h, \phi_{1}$ and $\phi_{2}$ are independent fields. Equations derived by extremizing the action (2.10) are Eq. (2.5) and

$$
\begin{aligned}
& \phi_{1}^{\prime \prime}+\frac{1}{r^{2}}\left(1-\phi_{1}^{2}-\phi_{2}^{2}\right) \phi_{1}+i \kappa \phi_{2}^{\prime}-h^{2} \phi_{1}=0 \\
& \phi_{2}^{\prime \prime}+\frac{1}{r^{2}}\left(1-\phi_{1}^{2}-\phi_{2}^{2}\right) \phi_{2}-i \kappa \phi_{1}^{\prime}-h^{2} \phi_{2}=0
\end{aligned}
$$

Eq. (3.1) and (3.2) are obtained by naively setting $A=0$ in (2.7) and (2.8). Eqs. (3.1) and (3.2) imply (2.9) but not necessarily (2.6). The left hand side of (2.6) does not vanish in general.

(ii) Unitary gauge $\left(\phi_{2}=0\right)$.

This gauge was employed in ref. [3]. Equations which follow are (2.5) and

$$
\begin{aligned}
& \phi_{1}^{2} A-\frac{i}{2} \kappa\left(1-\phi_{1}^{2}\right)=0 \\
& \phi_{1}^{\prime \prime}-A^{2} \phi_{1}+\frac{1}{r^{2}}\left(1-\phi_{1}^{2}\right) \phi_{1}-i \kappa A \phi_{1}=0
\end{aligned}
$$

Eq. (3.3) follows from (2.8) by setting $\phi_{2}=0$.

Eq. (3.3) is incompatible with the boundary condition (2.11) for $\kappa \neq 0$. Even for configurations which do not satisfy the Euler equations, the unitary gauge may not be possible. Suppose that a configuration $\left(h, \phi_{1}, \phi_{2}, A\right)$ satisfies the boundary condition (2.11), yielding a finite action. To bring it to the unitary gauge one has to choose $\tan f=\left(\phi_{2} / \phi_{1}\right)$ in (2.12). The action changes by a finite amount

$$
\left.\frac{4 \pi \kappa}{g^{2}} \tan ^{-1} \frac{\phi_{2}}{\phi_{1}}\right|_{r=0} ^{r=\infty} .
$$

The new $A$ satisfies the boundary condition only if $f^{\prime}=\left(\phi_{1} \phi_{2}^{\prime}-\phi_{1}^{\prime} \phi_{2}\right) /\left(\phi_{1}^{2}+\phi_{2}^{2}\right)$ vanishes at $r=\infty$, which is not generally satisfied.

(iii) Radiation gauge $\left(\partial_{\mu} A_{\mu}^{a}=0\right)$. 
The gauge condition implies that

$$
A^{\prime}+\frac{2}{r}\left(A-\frac{\phi_{2}}{r}\right)=0 \quad, \quad A(r)=\frac{2}{r^{2}} \int_{0}^{r} d r \phi_{2}(r)
$$

The action is viewed as a functional of $h, \phi_{1}$ and $\phi_{2}$. Equations derived are Eq. (2.5), (2.7) and

$$
\begin{aligned}
\left(\phi_{2}^{\prime}-A \phi_{1}\right)^{\prime}-A\left(\phi_{1}^{\prime}+A \phi_{2}\right)+\frac{\phi_{2}}{r^{2}}\left(1-\phi_{1}^{2}-\phi_{2}^{2}\right)-i \kappa\left(\phi_{1}^{\prime}+A \phi_{2}\right)-h^{2} \phi_{2} \\
-2 \int_{r}^{\infty} d u \frac{1}{u^{2}}\left\{\phi_{2} \phi_{1}^{\prime}-\phi_{1} \phi_{2}^{\prime}+A\left(\phi_{1}^{2}+\phi_{2}^{2}\right)+\frac{i}{2} \kappa\left(\phi_{1}^{2}+\phi_{2}^{2}-1\right)\right\}=0
\end{aligned}
$$

Here $A$ is expressed in terms of $\phi_{2}$ by (3.6).

There is residual gauge invariance. The gauge condition is respected by the transformation (2.12), provided $f(r)$ obeys the Gribov equation [11:

$$
f^{\prime \prime}+\frac{2}{r} f^{\prime}-\frac{2}{r^{2}}\left\{\phi_{1} \sin f+\phi_{2}(1-\cos f)\right\}=0
$$

or

$$
\ddot{f}+\dot{f}-2\left\{\phi_{1} \sin f+\phi_{2}(1-\cos f)\right\}=0 .
$$

Here a dot represents a derivative with respect to $t=\ln r$.

As was pointed out by Gribov, Eq. (3.9) is an equation for a point particle in a potential $V=2\left\{\phi_{1} \cos f-\phi_{2}(f-\sin f)\right\}$ with friction force. For $\phi_{1}=1$ and $\phi_{2}=0$, which corresponds to the trivial vacuum configuration $A_{\mu}^{a}=0$ in (2.3), there appear three types of solutions. With the initial condition $\left.f\right|_{r=0}=0$, (i) $f(r)=0$, (ii) $f(\infty)=\pi$, or (iii) $f(\infty)=-\pi$. The last two are the Gribov copies (see Fig. 1).

It is interesting to consider the relevance of the 't Hooft-Polyakov monopole configurations $\phi_{2}=0$ but $\phi_{1} \neq 0\left(\phi_{1}(0)=1\right.$ and $\phi_{1} \rightarrow 0$ as $\left.r \rightarrow \infty\right)$. In In terms of the Gribov equation, this case corresponds to a particle moving in a time-dependent potential. As the potential becomes exponentially small for large $t$, there can be a continuous family of solutions parameterized by the value of $f(\infty)$. The asymptotic value $f(\infty)$ depends of the

\footnotetext{
${ }^{1}$ Although this is clearly not a solution to the theory including the CS term, this exercise illustrates the possibility that summing of the Gribov copies may not lead to the cancellation of the monopole-type contribution.
} 


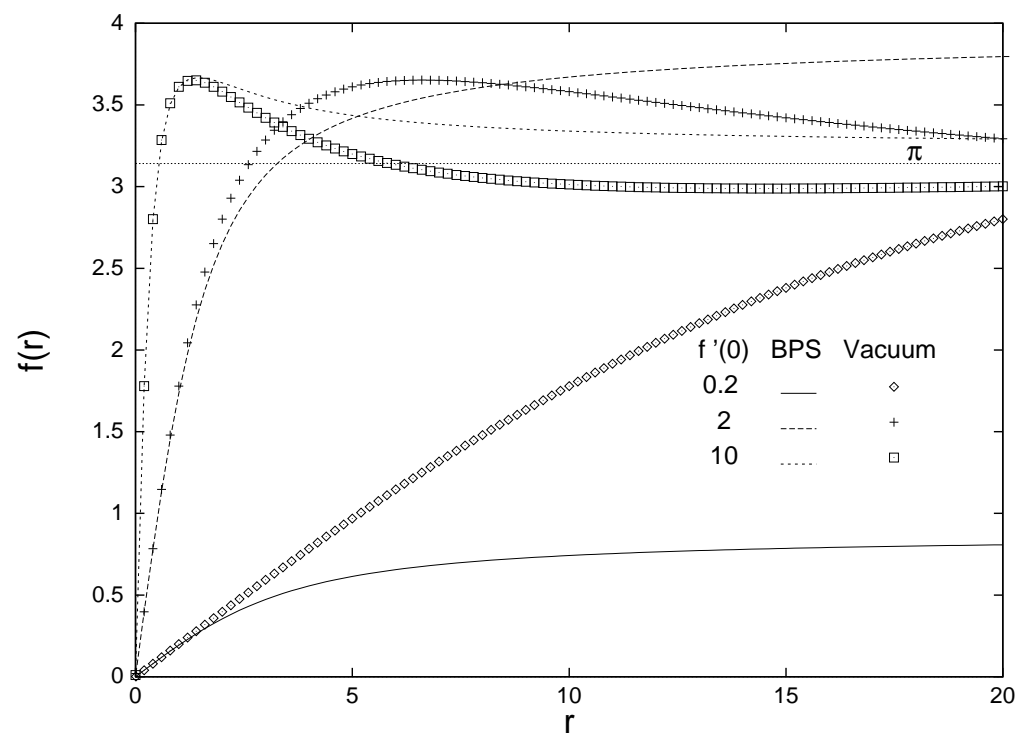

Figure 1: Solutions $f(r)$ to the Gribov Eq. (3.8). Solid lines correspond to the BPS monopoles with various values of $f^{\prime}(0)$ whereas points correspond to the vacuum. For the vacuum $f(\infty)=\pi$ for positive $f^{\prime}(0)$.

initial slope $f^{\prime}(0)$. In the BPS limit it ranges from 0 to 3.98. See Fig. 2. For $\left|f^{\prime}(0)\right| \ll 1$, $|f(r)|$ remains small. For $|f(r)| \gg 1, f(r)$ approaches an asymptotic value before $\phi_{1}$ and $\phi_{2}$ make sizable changes, i.e., $f(r)$ behaves as in the vacuum case. The maximum value for $|f(\infty)|$ is attained for $f^{\prime}(0)= \pm 2.62$.

These Gribov copies in the radiation gauge lead to an important consequence in the Chern-Simons theory. Under the gauge transformation the action changes as

$$
S \rightarrow S+i \frac{4 \pi \kappa}{g^{2}}(f(\infty)-f(0))
$$

Recall that the Chern-Simons coefficient is quantized, i.e. $4 \pi \kappa / g^{2}$ is an integer. If $f(\infty)$ takes an arbitrary value, or takes all values in the interval $[-\pi,+\pi]$, then the integration over the parameter $f(\infty)$ in the path integral could kill contribution coming from the monopole configuration as argued by Affleck et. al. [5].2 A close examination of Eq. (3.8) reveals that this is not the case. As mentioned above, $f(\infty)$ for the 't Hooft-Polyakov

\footnotetext{
${ }^{2}$ In ref. [5], the parameter $f(\infty)$ is interpreted as the collective coordinate of the monopole configuration, and is therefore integrated over. Here, we alternatively associate $f(\infty)$ with the Gribov copies within a given gauge, corresponding to the solutions of eq. (3.8). In this interpretation, one sums over the Gribov copies, i.e., one integrates over the parameter $f(\infty)$.
} 


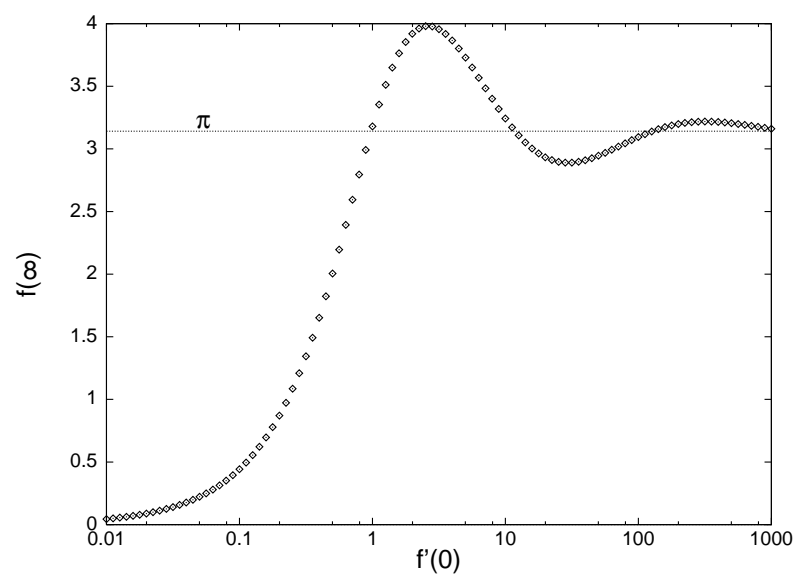

Figure 2: $f(\infty)$ for BPS monopole vs. initial slope $\left(f^{\prime}(0)\right)$.

monopole ranges from -3.98 to 3.98 , depending on $f^{\prime}(0)$. There seems no reason for expecting the cancellation.

\section{(iv) Temporal gauge $\left(A_{3}^{a}=0\right)$}

This gauge destroys spherical symmetry in the Euclidean space, but allows physical interpretation in the Minkowski spacetime.

We are going to show that there are complex monopole solutions in certain gauges. We argue also that in the case of the radiation gauge Gribov copies do not lead to cancellation. When $\phi_{2}$ is complex in (3.8), a solution $f(r)$ to Eq. (3.8) necessarily becomes complex. In other words the transformation specified with $f(r)$ is not a gauge transformation. However, one can show that there are solutions $f(r)$ to Eq. (3.8) in which $f(0)=0$ and $f(\infty)$ is real.

In terms of a gauge invariant quantity

$$
\begin{aligned}
\eta & =\left(\phi_{1}+i \phi_{2}\right) e^{-i \int^{r} A(r) d r} \\
\eta^{*} & =\left(\phi_{1}-i \phi_{2}\right) e^{+i \int^{r} A(r) d r}
\end{aligned}
$$

the action (2.10) simplifies to

$$
\begin{gathered}
S=\frac{4 \pi}{g^{2}} \int_{0}^{\infty} d r\left\{\eta^{\prime *} \eta^{\prime}+\frac{1}{2 r^{2}}\left(1-\eta \eta^{*}\right)^{2}-\frac{\kappa}{2}\left(\eta^{\prime} \eta^{*}-\eta \eta^{\prime *}\right)+i \kappa A\right. \\
\left.+\frac{r^{2}}{2}\left(h^{\prime}\right)^{2}+h^{2} \eta \eta^{*}+\frac{\lambda r^{2}}{4}\left(h^{2}-v^{2}\right)^{2}\right\} .
\end{gathered}
$$


Note that $\int_{0}^{\infty} d r A(r)$ implicitly depends on $\eta$ and $\eta^{*}$.

\section{Complex monopole solutions}

As discussed in the previous section, the gauge is fixed in quantum theory. The aim of this section is to show the existence of complex monopole solutions in certain gauges. We shall see a subtle relation among different gauges. A solution in one gauge can be transformed into another gauge by a "complex" gauge transformation, but the transformed configuration may not be a solution in the new gauge. This is a reflection of the gauge noninvariance of the original theory in the presence of the Chern-Simons term. It implies that relevant field configurations in the path integral may appear different, depending on the gauge. This raises a question if all gauges are equivalent as they should. A related issue has been analyzed in the temporal gauge in ref. [12]. It has been shown that implementation of constraints before quantization does not yield the correct physics in Chern-Simons theory.

We note that the t'Hooft $U(1)$ field strength is defined by

$$
F_{\mu \nu}=\frac{h^{a}}{h} F_{\mu \nu}^{a}-\frac{1}{h^{3}} \epsilon_{a b c} h^{a}\left(D_{\mu} h\right)^{b}\left(D_{\nu} h\right)^{a}
$$

With our ansatz it becomes

$$
F_{\mu \nu}=-\frac{\epsilon_{\mu \nu a} \hat{x}^{a}}{r^{2}}
$$

It is independent of the details of $\left(\phi_{1}, \phi_{2}, A\right)$. In the solution for $\kappa \neq 0, \phi_{2}$ and therefore non-abelian field strengths generally become complex. However, the 't Hooft tensor (4.2) remains real with the standard Abelian field strength representing a magnetic monopole. We call the solution a complex monopole. We also recall that in the BPS limit $\lambda=\kappa=0$ the solution is given by

$$
h(r)=v \operatorname{coth}(v r)-\frac{1}{r} \quad, \quad \phi_{1}(r)=\frac{v r}{\sinh (v r)} \quad, \quad \phi_{2}=A=0
$$

(i) Radial gauge $(A=0)$ 
In this gauge there is no Gribov copy. Equations to be solved are Eqs. (2.5), (3.1), and (3.2). There is a solution in which $h$ and $\phi_{1}$ are real, but $\phi_{2}$ is pure imaginary. An appropriate ansatz is

$$
\phi_{1}(r)=\zeta(r) \cosh \frac{\kappa r}{2} \quad, \quad \phi_{2}(r)=i \zeta(r) \sinh \frac{\kappa r}{2}
$$

The non-Abelian field strengths are

$$
\begin{aligned}
F_{\mu \nu}^{a}=\frac{1}{r^{2}} \epsilon_{\mu \nu b} \hat{x}^{a} \hat{x}^{b}\left(\zeta(r)^{2}-1\right) & +\frac{\kappa}{2 r}\left(\epsilon_{a \mu \nu}-\epsilon_{\mu \nu b} \hat{x}^{a} \hat{x}^{b}\right) \zeta(r) \sinh \frac{\kappa r}{2} \\
& +\frac{i \kappa}{2 r}\left(\delta^{a \nu} \hat{x}^{\mu}-\delta^{a \mu} \hat{x}^{\nu}\right) \zeta(r) \cosh \frac{\kappa r}{2}
\end{aligned}
$$

The three equations reduce to

$$
\begin{aligned}
& \frac{1}{r^{2}} \frac{d}{d r}\left(r^{2} \frac{d}{d r} h\right)-\lambda\left(h^{2}-v^{2}\right) h-\frac{2}{r^{2}} \zeta(r)^{2} h=0 \\
& \zeta^{\prime \prime}-\frac{1}{r^{2}}\left(\zeta^{2}-1\right) \zeta-\left(h^{2}+\frac{\kappa^{2}}{4}\right) \zeta=0
\end{aligned}
$$

The boundary conditions are (a) $h=0$ and $\zeta=1$ at $r=0$ and (b) $h=v$ and $e^{\kappa r / 2} \zeta=0$ at $r=\infty$. Eq. (4.6) follows by minimizing the integral

$$
S=\int_{0}^{\infty} d r\left\{\left(\zeta^{\prime}\right)^{2}+\frac{\kappa^{2}}{4} \zeta^{2}+\frac{1}{2 r^{2}}\left(\zeta^{2}-1\right)^{2}+\zeta^{2} h^{2}+\frac{r^{2}}{2}\left(h^{\prime}\right)^{2}+\frac{\lambda r^{2}}{4}\left(h^{2}-v^{2}\right)^{2}\right\}
$$

The solution can be easily found numerically, as it follows from the minimization of (4.7). It has a finite Euclidean action (2.10) and is depicted in Fig. 3.

\section{(ii) Unitary gauge $\left(\phi_{2}=0\right)$}

As remarked in the previous section there is no monopole solution with a finite action in this gauge. It may be of interest to investigate a configuration which is related to the complex monopole solution in the radial gauge by a "complex" gauge transformation. As $\phi_{2} / \phi_{1}=i \tanh (\kappa r / 2)$ is pure imaginary in the radial gauge, the gauge function in (2.12), which brings the solution in the unitary gauge, is $f=i \kappa r / 2$. Hence $A^{\text {unitary }}=-i \kappa / 2$, which gives a divergent action. Of course the configuration thus obtained does not satisfy the equations in the unitary gauge. 


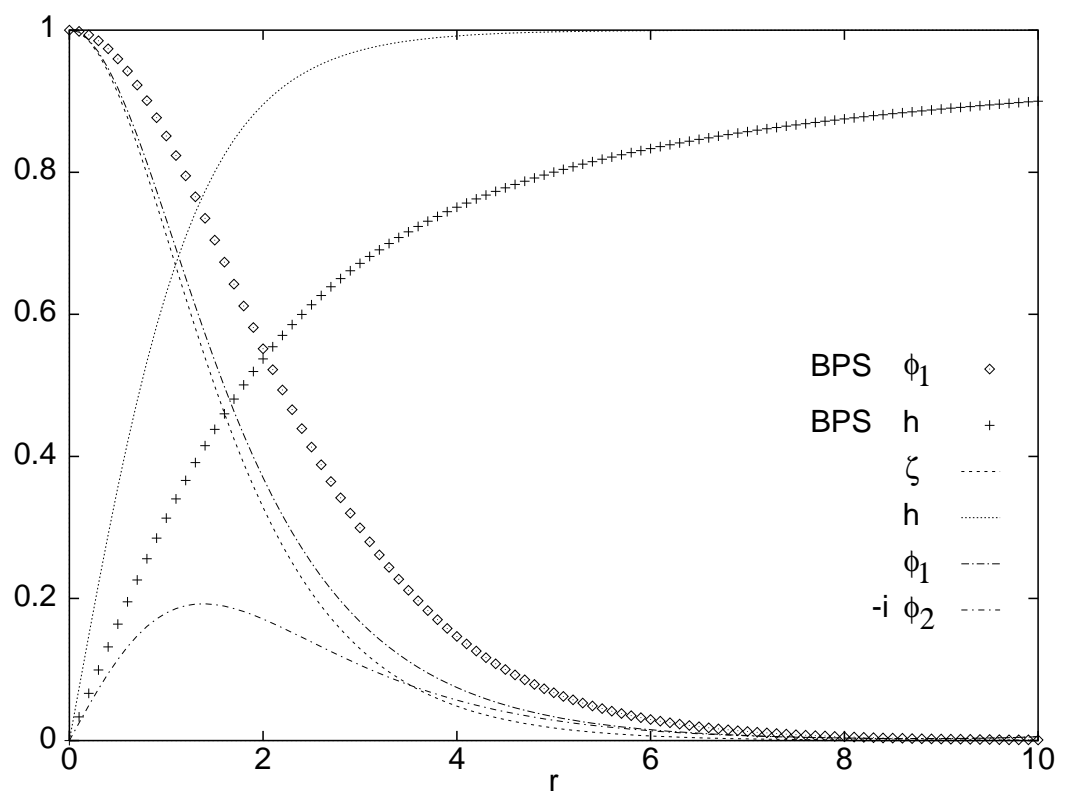

Figure 3: The solution in the radial gauge for $v=1, \kappa=0.5$ and $\lambda=0.5$. The solutions at arbitrary values of $\kappa$ and $\lambda$ are numerically generated using the exact known BPS solutions for $\phi_{1}$, BPS and $h_{B P S}$. In this plot we show the solutions for $\zeta(r)$ and $h(r)$, and also $\phi_{1}(r)$ and $-i \phi_{2}(r)$.

(iii) Radiation gauge $\left(\partial_{\mu} A_{\mu}^{a}=0\right)$

Again we look for a solution in which $\phi_{2}$ is pure imaginary: $\phi_{2}=i \bar{\phi}_{2}$ and $A=i \bar{A}$. Equations to be solved are

$$
\begin{aligned}
& \frac{1}{r^{2}} \frac{d}{d r} r^{2} \frac{d}{d r} h-\lambda\left(h^{2}-v^{2}\right) h-\frac{2}{r^{2}}\left(\phi_{1}^{2}-\bar{\phi}_{2}^{2}\right) h=0 \\
& \frac{d}{d r}\left(\begin{array}{c}
D \phi_{1} \\
D \bar{\phi}_{2}
\end{array}\right)-\left(\bar{A}+\frac{\kappa}{2}\right)\left(\begin{array}{c}
D \bar{\phi}_{2} \\
D \phi_{1}
\end{array}\right)-\left\{\frac{1}{r^{2}}\left(\phi_{1}^{2}-\bar{\phi}_{2}^{2}-1\right)+h^{2}+\frac{\kappa^{2}}{4}\right\}\left(\begin{array}{c}
\phi_{1} \\
\bar{\phi}_{2}
\end{array}\right) \\
& -\left(\begin{array}{c}
0 \\
1
\end{array}\right) \int_{r}^{\infty} d u \frac{2}{u^{2}}\left\{\bar{\phi}_{2} D \phi_{1}-\phi_{1} D \bar{\phi}_{2}-\frac{\kappa}{2}\right\}=0
\end{aligned}
$$

where

$$
\left(\begin{array}{c}
D \phi_{1} \\
D \bar{\phi}_{2}
\end{array}\right)=\left(\begin{array}{c}
\phi_{1}^{\prime} \\
\bar{\phi}_{2}^{\prime}
\end{array}\right)-\left(\bar{A}+\frac{\kappa}{2}\right)\left(\begin{array}{c}
\bar{\phi}_{2} \\
\phi_{1}
\end{array}\right) \quad, \quad \bar{A}=\frac{2}{r^{2}} \int_{0}^{r} d u \bar{\phi}_{2}(u)
$$

It is not easy to find a simple ansatz for $\phi_{1}$ and $\bar{\phi}_{2}$ which solves the equations.

Note that the equations in (4.8) are in a gauge covariant form except for the last term in the second equation. If one makes a complex gauge transformation, promoting $f$ in 
(2.12) to a complex $f=i \bar{f}$,

$$
\left(\begin{array}{c}
\phi_{1} \\
\bar{\phi}_{2}
\end{array}\right) \rightarrow\left(\begin{array}{ll}
+\cosh \bar{f} & -\sinh \bar{f} \\
-\sinh \bar{f} & +\cosh \bar{f}
\end{array}\right)\left(\begin{array}{c}
\phi_{1} \\
\bar{\phi}_{2}
\end{array}\right) \quad, \quad \bar{A} \rightarrow \bar{A}-\bar{f}^{\prime}
$$

then the equations remain the same except that the matrix factor $(0,1)$ in the second equation in $(4.8)$ is replaced by $(\sinh \bar{f}, \cosh \bar{f})$. Even if one chooses $\bar{f}$ such that the new $\bar{A}=0$, the last term remains. The transformed equations are different from the equations in the radial gauge.

The solution for Eqs. (4.8) and (4.9) need to be found numerically, which is left for future work. Let us suppose that there is a solution. One has to ask if the solution is unique. We explore "complex" Gribov copies of the solution. As $\phi_{2}$ is pure imaginary, solutions to (3.8) necessarily become complex. There is no "real" Gribov copy. With $f=f_{R}+i f_{I}$ Eq. (3.8) becomes

$$
\begin{aligned}
& f_{R}^{\prime \prime}+\frac{2}{r} f_{R}^{\prime}-\frac{2}{r^{2}}\left\{\phi_{1} \sin f_{R} \cosh f_{I}-\bar{\phi}_{2} \sin f_{R} \sinh f_{I}\right\}=0 \\
& f_{I}^{\prime \prime}+\frac{2}{r} f_{I}^{\prime}-\frac{2}{r^{2}}\left\{\phi_{1} \cos f_{R} \sinh f_{I}+\bar{\phi}_{2}\left(1-\cos f_{R} \cosh f_{I}\right)\right\}=0 .
\end{aligned}
$$

Boundary conditions are $f_{R}(0)=f_{I}(0)=0$ and $f_{R}(\infty), f_{I}(\infty)=$ finite. Although the meaning of these solutions is not clear for $f_{I}(r) \neq 0$, we point out that solutions satisfying $f_{I}(\infty)=0$ might have special role in the path integral. In view of (2.14), such copies carry the additional oscillatory factor $\left(4 \pi i \kappa / g^{2}\right) f_{R}(\infty)$ in the path integral. Examination of Eq. (4.11) with representative $\phi_{1}$ and $\bar{\phi}_{2}$ shows that such a solution is uniquely found with given $f_{R}^{\prime}(0) . f_{R}(\infty)$ is determined as a function of $f_{R}^{\prime}(0)$. The range of $f_{R}(\infty)$ is not restricted to $[-\pi, \pi]$. No cancellation is expected in the path integral from these copies.

\section{Remarks on other works}

In this section, we wish to point out some differences between our work and ones found in the earlier literature, especially as there appears to be some confusion about the relevance of monopole configurations in the path integral.

(i) Pisarski's work 
In ref. [3], the unitary gauge $\phi_{2}=0$ was adopted. As remarked before, the boundary conditions cannot be satisfied in this gauge for $\kappa \neq 0$. In other words, solutions obtained in this gauge after relaxing the boundary conditions necessarily have an infinite action for $\kappa \neq 0$. This is what Pisarski found. If one considers a monopole-antimonopole pair, the action can be made finite. The action is proportional to the distance between the pair, which leads to the confinement picture of monopole-antimonopole pairs.

We also remark again that Pisarski's solution in the unitary gauge is different from the configuration obtained from the solution in the radial gauge in Section 3-(i) by a gauge transformation.

(ii) Affleck, Harvey, Palla, and Semenoff's work

It has been recognized in ref. [5] that gauge copies of monopole solutions can lead to cancellation in the path integral. As expressed in (2.14), gauge copies yield an extra factor $\exp \left\{\left(4 \pi i \kappa / g^{2}\right) f(\infty)\right\}=\exp \{i n f(\infty)\}$ where the quantization condition for $\kappa$ has been employed. In ref. [5] the factor $f(\infty)$ was promoted to a collective coordinate $\Lambda$. It was argued then that the integration over the collective coordinate $\Lambda$ from 0 to $2 \pi$ gives a vanishing contribution when the monopole number is non-vanishing.

However, this argument is incomplete. As explained in Section 2-(iii), the possible range for $f(\infty)$ in the radiation gauge for the monopole in the BPS limit is $[-3.98,3.98]$. No cancellation is expected.

(iii) Fradkin and Schaposnik's work

In ref. [6], the authors start with a gauge invariant theory with massive fermions in the Abelian theory. The integration of fermion degrees induces a Chern-Simons term, which leads to the deconfinement of charges. The authors have argued that there appears a linear confining potential between monopoles and antimonopoles so that a monopole gas becomes a dipole gas exhibiting no Debye screening and destroying the confinement picture. Further, they argue that the lack of the gauge invariance of the Chern-Simons term makes gauge copies of monopole configurations leads to the cancellation in the path integral. 
Their argument is unsatisfactory in the context of our work in two respects. First they consider monopole configurations obtained in the absence of the Chern-Simons term and insert them into the Chern-Simons term to find implications. The fact that the configurations do not solve the equations in the presence of the Chern-Simons term leads to a linear potential among monopoles and antimonopoles. This argument is not consistent; one should examine solutions in the full theory including the Chern-Simons term.

Secondly their argument is given for the Abelian theory with monopole configurations carrying singularity at their cores. Although the authors claim that their argument goes through for the non-Abelian case as well, there are important differences between the two cases. In the Abelian theory with monopole background fields, the Chern-Simons term, after a gauge transformation $\omega(\vec{x})$, yields an extra factor $\exp \left\{i \sum_{a} n_{a} \omega\left(\vec{x}_{a}\right) / 2\right\}$ where $n_{a}$ and $\vec{x}_{a}$ are magnetic charge and position of the $a$-th monopole. Hence Fradkin and Schaposnik argue that the integration of $\omega(\vec{x})$ eliminates contributions of monopoles completely. The factor $\omega\left(\vec{x}_{a}\right)$ results from the singularity of the Abelian monopole configuration at $\vec{x}=\vec{x}_{a}$. In the non-Abelian theory, however, monopole configurations are regular everywhere and the Chern-Simons term produces a factor $\exp \{\inf (\infty)\}$ (see (2.14)). Only the value of the gauge potential $f(r)$ at $r=\infty$ is important. We have observed that $f(\infty)$ takes values in a limited range and no cancellation is expected.

(iv) Diamantini, Sodano and Trugenberger's work

Diamantini et al. have formulated the compact Maxwell-Chern-Simons theory in the dual theory on the lattice, in which the dual field variable $f^{\mu}=\epsilon^{\mu \nu \rho} F_{\nu \rho} / 2$ becomes fundamental. [7] In this compact $U(1)$ theory monopoles naturally arise on the lattice. Diamatini et al. have found a complex solution for a monopole-antimonopole pair. The dual field $f^{\mu}$ has a string singularity between the two poles. In the presence of the Chern-Simons term the string, carrying a magnetic flux, becomes electrically charged. As Henneaux and Teitelboim pointed out, 13] such a string becomes observable and has a finite energy density so that the monopole-antimonopole pair is linearly confined.

Although the confinement picture of monopoles in ref. [7] is consistent with refs. [3, 5, 6], due caution is necessary in extending the picture to non-Abelian theory. The dual 
theory in [7] is entirely Abelian, consisting of only gauge fields. In the Georgi-GlashowChern-Simons model, the solution is regular everywhere. There is no place where an observable string singularity enters. It is not clear if the role of the Higgs field in the continuum non-Abelian theory can be completely mimiced by the lattice structure in the dual theory.

(v) Jackiw and Pi's work

Jackiw and Pi [14 have argued that the addition of the Chern-Simons interaction destroys the topological excitations such as monopoles. They parameterize $\phi_{1}=\rho \cos \theta$ and $\phi_{2}=\rho \sin \theta$. The boundary conditions (2.11) are $\rho(0)=1, \theta(0)=1$ and $\rho(\infty)=0$. Under a gauge transformation (2.12), $\theta \rightarrow \theta-f$ and $A \rightarrow A-f^{\prime} . \theta^{\prime}-A$ is gauge invariant.

The authors employ one of the classical equations of motion, Eq. (2.6), to reduce the action, which is subsequently minimized. However, as remarked near the end of Section 2, Eq. (2.6) is incompatible with the boundary condition ensuring the finiteness of the action. Consequently all monopole configurations have an infinite action in their formalism.

Indeed, Eq. 2.6) reads $\rho^{2}\left(\theta^{\prime}-A-\frac{1}{2} i \kappa\right)=-\frac{1}{2} i \kappa$. Upon utilizing this equation, the effective action $(2.10)$ is reduced to

$$
\begin{aligned}
S_{J P}=\frac{4 \pi}{g^{2}} \int_{0}^{\infty} d r & \left\{\rho^{\prime 2}+\frac{\kappa^{2}}{4}\left(\rho^{2}+\frac{1}{\rho^{2}}\right)\right. \\
& \left.+\frac{1}{2 r^{2}}\left(\rho^{2}-1\right)^{2}+\frac{r^{2}}{2} h^{\prime 2}+h^{2} \rho^{2}+\frac{\lambda r^{2}}{4}\left(h^{2}-v^{2}\right)^{2}\right\}
\end{aligned}
$$

The presence of the $1 / \rho^{2}, h^{2} \rho^{2}$, and $r^{2}\left(h^{2}-v^{2}\right)^{2}$ terms makes it impossible to have a configuration of a finite action.

In quantum theory a gauge is fixed. In the radial gauge $A=0$, for instance, one of the classical equation, Eq. (2.6), which is derived by varying $A$, does not follow. Hence the relation $\rho^{2}\left(\theta^{\prime}-A-\frac{1}{2} i \kappa\right)=-\frac{1}{2} i \kappa$ should not be used to simplify the action.

(vi) More subtleties

There remains subtle delicacy in defining the quantum theory of the Chern-Simons theory. We have started with the Faddeev-Popov formula (2.15). If one picks a radial gauge $F(A)=x^{\mu} A_{\mu}^{a}=0$, then there is a complex monopole solution which extremizes 
the restricted action, namely the action in the given gauge slice. What happens, say, in the temporal gauge? The answer is not clear. As explained in Section 4, solutions look different, depending on the gauge chosen. Two operations in the path integral, fixing a gauge and finding configurations which extremize the action, do not commute with each other in the Chern-Simons theory. In the above papers by Pisarski and by Jackiw and Pi the action is extremized with respect to arbitrary variations of gauge fields, and then a gauge is picked. This procedure yields one more equation to be solved, and in general this equation turns out incompatible with the finiteness of the action for monopoles.

In QED or QCD the order of the two operations does not matter. For instance, instanton solutions in QCD can be found in any gauge. The apparent noncommutability of the two operations in the Chern-Simons theory is traced back to the gauge non-invariance of the Chern-Simons term particularly in the monopole background. In the original derivation

of the Faddeev-Popov formula (2.15) the gauge invariance of the action was assumed. The formula (2.15) needs to be scrutinized in the Chern-Simons theory. There is also ambiguity in the definition of the Chern-Simons term as pointed out by Deser et al. [18 Further investigation is necessary.

\section{Chern-Simons-Higgs theory}

In the absence of Yang-Mills term one obtains the Chern-Simons-Higgs theory. Although the pure Chern-Simons theory defines a topological field theory, one obtains a dynamical theory after matter couplings are introduced. The equations of motion for the gauge fields are first order in derivatives which makes the theory easier to handle at least at the classical level. The issue whether this theory makes sense or not at the quantum level was addressed by Tan et. al. in ref. [15]. Using the two loop effective potential in the dimensional regularization scheme, it has been shown that one has to start with the Yang-Mills term, but the limit of vanishing Yang-Mills term exists after renormalization. In this section we are mainly interested in the Chern-Simons-Higgs theory at the tree level.

Monopoles in CS-Higgs theory was discussed in references [16] and [17]. Lee showed that instantons of an infinite action induce an effective vertex which break the global 
part of the $U(1)$ gauge symmetry and lead to non-conservation of charge. Edelstein and Schaposnik showed that there are no monopole solutions of a finite action. Using the vacuum equations of motion they showed that magnetic field is everywhere orthogonal to the Higgs field in the isospin space in contrast to the 't Hooft-Polyakov hedgehog solution where two fields are parallel. This orthogonality of the magnetic field and Higgs field forces the $U(1)$ field strength to vanish for finite action monopoles. In this section we are going to reproduce the same conclusions as in [17] in a way that parallels our discussions in the previous parts of this paper.

The action in terms of the monopole ansatz is

$$
\begin{aligned}
S=\frac{4 \pi}{g^{2}} \int_{0}^{\infty} d r\{ & +i \kappa\left[\phi_{1}^{\prime} \phi_{2}-\phi_{2}^{\prime}\left(\phi_{1}-1\right)+A\left(\phi_{1}^{2}+\phi_{2}^{2}-1\right)\right] \\
& \left.+\frac{r^{2}}{2} h^{\prime 2}+h^{2}\left(\phi_{1}^{2}+\phi_{2}^{2}\right)+\frac{\lambda r^{2}}{4}\left(h^{2}-v^{2}\right)^{2}\right\}
\end{aligned}
$$

The finiteness of the action demands boundary conditions $h=v, \phi_{1}=\phi_{2}=A=0$ at $r=\infty$, but imposes no condition at $r=0$. The regularity of the configuration at the origin requires $h=\phi_{2}=0, \phi_{1}=1$ at $r=0$.

If all $A, \phi_{1}, \phi_{2}$ and $h$ are regarded as independent variables, then equations of motion are

$$
\begin{aligned}
& \phi_{1}^{2}+\phi_{2}^{2}-1=0 \\
& i \kappa\left(\phi_{2}^{\prime}-A \phi_{1}\right)-h^{2} \phi_{1}=0 \\
& i \kappa\left(\phi_{1}^{\prime}+A \phi_{1}\right)+h^{2} \phi_{2}=0 \\
& \left(r^{2} h^{\prime}\right)^{\prime}-2\left(\phi_{1}^{2}+\phi_{2}^{2}\right) h-\lambda\left(h^{2}-v^{2}\right) r^{2} h=0 .
\end{aligned}
$$

Clearly the first equation (6.2) is incompatible with the boundary conditions at $r=0$ and $\infty$

However, a gauge is fixed in quantum theory. Take the radial gauge $A=0$. Then equations of motion are (6.3), (6.4), and (6.5) where $A$ is set to be 0 . In the Jackiw-Pi parameterization $\phi_{1}+i \phi_{2}=\rho e^{i \theta}$, Eqs. (6.3) and (6.4) become

$$
\left(i \kappa \theta^{\prime}+h^{2}\right) \rho=0 \quad, \quad i \kappa \rho^{\prime}=0
$$


The second equation implies that $\rho$ is constant, which conflicts with the boundary conditions. Hence there is no regular monopole configuration of a finite action. In fact equations of motion totally eliminate the spherically symmetric gauge fields and the resulting action is that of a Higgs field alone with the action given by:

$$
S=\frac{4 \pi}{g^{2}} \int_{0}^{\infty} d r\left\{\frac{r^{2}}{2} h^{\prime 2}+\frac{\lambda r^{2}}{4}\left(h^{2}-v^{2}\right)^{2}\right\}
$$

\section{Conclusions and discussions}

We have seen that in the Georgi-Glashow-Chern-Simons theory, complex monopoles exist, and that they have a non-vanishing contribution to the path integral. As we have shown, the cleanest way to see this is in the radial gauge. The action is minimized by complex solutions, and is real and finite. Furthermore, the solutions have the usual characteristics of monopoles. They have $U(1)$ field strength given by $F_{\mu \nu}=-\epsilon_{\mu \nu} \hat{x}^{a} / r^{2}$ and mass $\sim 1 / g^{2}$. As a consequence, the long range order in the Higgs vacuum is destroyed. However, we must recognize that we are far from understanding this theory at quantum level, or beyond semiclassical approximation. The understanding of the quantum theory is obscured by the gauge non-invariance of the Chern-Simons term.

We started with a theory with compact U(1) symmetry, where by definition the gauge transformation parameter (which we called $f$ ) is originally a real-valued function. However, in discussing the Gribov copy problem in the radiation gauge, we have looked for complex field configurations which are related to each other by complex $f$.

Curiously enough, the gauge invariant part of the action (i.e., everything other than the CS term) is still invariant under the transformation with complex $f$. At the tree level there are many saddle points in the complex field configuration space which are related by these complex f's. At the one loop level, however, the effective action would not be invariant under complex $f$ transformations. The physical interpretation of $f$ being complex is not obvious at all. 
If we had restricted the gauge parameter $f$ in this theory to be real and not allow complex $f$, then the absence of real solutions to the Gribov equation in the radiation gauge would lead to no Gribov copies.

We have adopted the view that complex solutions to Gribov's equation correspond to generalized Gribov copies of complex saddle points. We understand that this is a question not completely settled, and warrants further investigation. Meanwhile we conclude that within our semi-classical approximation, it appears that summation over the Gribov copies (or integrating over the collective coordinates) of the complex monopole solutions does not lead to the cancellation of the monopole contribution. What if quantum corrections to the Jacobian of the Gribov copies somehow cancel the effect after all? This is one of the questions which we can not answer in this model unless we learn how to go beyond the semi-classical approximation. We remark that one could raise the same objection for the real monopole case where it has been argued in the literature that the integral cancels. Recall that the issues of the non-invariance of the CS term, and the problems associated with the quantization are irrespective of whether the monopole is real or complex.

In closing, let us remark that complex gauge field configurations have been studied before in the literature [19]-22]. In particular Wu and Yang have given a prescription of how complex gauge fields in $S U(2)$ theory can be converted to real gauge fields for the group $S L(2, C)$. Witten describes a way to quantize theories with non-compact gauge groups. He shows that Chern-Simons theory with the group $S L(2, C)$ is equivalent to the $2+1$ dimensional quantum gravity. According to the analysis of ref. [20], one can obtain the Einstein-Hilbert theory both in the de-Sitter and anti-de-Sitter spaces. Further research is needed to understand how one can generalize this description to the space where cosmological constant is zero and how one can couple matter to gravity in this language. Making contact between these three-dimensional gravity related considerations and the present work is the subject of a separate study to be presented elsewhere.

\section{Acknowledgements}


The authors would like to thank Bob Pisarski and Pierre van Baal for useful discussions. They are also grateful to Roman Jackiw and So-Young Pi for many enlightening remarks and for pointing out an error in the original version of the paper. This work was supported in part by the U.S. Department of Energy under contracts DE-FG02-94ER40823. One of the authors (Y.H.) would like to thank the Yukawa Institute for Theoretical Physics for its hospitality where the final part of the work was done in summer 1998.

\section{References}

[1] A. M. Polyakov, Phys. Lett. B59 (1975) 80; Nucl. Phys. B120 (1977) 429.

[2] S. Deser, R. Jackiw and S. Templeton, Phys. Rev. Lett. 48 (1982) 976.

[3] R. D. Pisarski, Phys. Rev. D34 (1986) 3851.

[4] E. D. D'Hoker and L. Vinet, Ann. Phys. (N.Y.) 162 (1985) 413.

[5] I. Affleck, J. Harvey, L. Palla and G. Semenoff, Nucl. Phys. B328 (1989) 575.

[6] E. Fradkin and F. A. Schaposnik, Phys. Rev. Lett. 66 (1991) 276.

[7] M. C. Diamantini, P. Sodano and C. A. Trugenberger, Phys. Rev. Lett. 71 (1993) 1969.

[8] Y. Hosotani, Phys. Lett. B69 (1977) 499.

[9] M. C. Diamantini, P. Sodano and C. A. Trugenberger, Nucl. Phys. B474 (1996) 641.

[10] G. 't Hooft, Nucl. Phys. B79 (1974) 276.

[11] V. N. Gribov, Nucl. Phys. B139 (1978) 1.

[12] G. V. Dunne, R. Jackiw and C. A. Trugenberger, Ann. Phys. (N.Y.) 194 (1989) 197.

[13] M. Henneaux and C. Teitelboim, Phys. Rev. Lett. 56 (1986) 689.

[14] R. Jackiw and S.Y. Pi, Phys. Lett. B423 (1998) 364

[15] P-N. Tan, B. Tekin and Y. Hosotani, Phys. Lett. B388 (1996) 611; Nucl. Phys. B502 (1997) 483.

[16] K. Lee, Nucl. Phys. B373 (1992) 735.

[17] J. D. Edelstein and F. A. Schaposnik, Nucl. Phys. B425 (1994) 137.

[18] S. Deser, L. Griguolo and D. Seminara, preprint hep-th/9712132.

[19] T T. Wu and C.N. Yang, Phys. Rev. D13 (1976) 3233.

[20] E. Witten, Nucl. Phys. B311 (1988) 46; Com. Math. Phys.137 (1991) 29.

[21] C. H. Oh , L.C. Sia and E. C. G. Sudarshan, Phys. Lett. B248 (1990) 295.

[22] M. Asorey, F. Falceto, J. L. Lopez and G. Luzon, Phys. Lett. B349 (1995) 125. 